\title{
Assessment and management of pediatric pain based on the opinions of health professionals
}

\author{
Maria Beatriz Martins Linhares ${ }^{1}$, Nátali C.A.C. Oliveira ${ }^{1}$, Fernanda N.P. Doca ${ }^{2}$, Francisco E. \\ Martinez ${ }^{1}$, Ana Paula P. Carlotti ${ }^{1}$, and Gordon Allen Finley ${ }^{3}$ \\ 1. Universidade de São Paulo, Ribeirão Preto, SP, Brazil \\ 2. Universidade de Brasília, Brasília, DF, Brazil \\ 3. University of Dalhousie, Halifax, NS, Canada
}

\begin{abstract}
The present study characterized the opinions of health professionals about strategies for assessing and managing pediatric pain in a public teaching hospital. The sample consisted of 92 health professionals who worked in pediatric wards, pediatric intensive care, and neonatal intensive care. The sample included 45 doctors, 18 nurses, 16 psychologists, eight physiotherapists, and five occupational therapists. Data were collected through a self-administered questionnaire that included 22 open questions on the following topics: pain assessment, pharmacological management, and non-pharmacological interventions. Each area was analyzed with regard to actions, resources, gaps, and needs. The questionnaire was developed based on the principles of Strategic Planning. Two trained researchers analyzed the thematic content of all of the responses. With regard to actions and resources, $33 \%$ of the respondents mentioned the use of instruments for pain assessment, $73 \%$ reported that they prescribed pharmacological treatments for symptoms of pain, and $26 \%$ reported the use of non-pharmacological interventions for the relief of pain. The professionals predominantly reported a lack of training for pain assessment and management, standardized protocols, and human and material resources. Consequently, $96 \%$ of the professionals reported the necessity for educational training and standardized implementation guidelines for pain assessment services. These findings provide a baseline of the health professional's opinions of pain issues, which are essential for implementing and increasing pain assessment and management policies institutionally. Keywords: pediatric pain, hospitalization, healthcare professionals, assessment, management.
\end{abstract}

Received 08 March 2013; received in revised form 29 June 2013; accepted 01 July 2013. Available online 07 March 2014.

\section{Introduction}

Pain constitutes a health problem, the relief and treatment of which are recognized as human rights by several organizations, especially the World Health Organization and International Association for the Study of Pain (1997). Despite increasing scientific evidence

Maria Beatriz Martins Linhares, Department of Neurosciences and Behavior, Ribeirão Preto Medical School, University of São Paulo. Nátali C.A.C. Oliveira, Hospital of Clinics, Ribeirão Preto Medical School, University of São Paulo, and master's degree in Mental Health Program, Ribeirão Preto Medical School, University of São Paulo. Fernanda N.P. Doca, Brasília University Hospital and Distrito Federal Base Hospital, Institute of Psychology, University of Brasília. Francisco E. Martinez and Ana Paula P. Carlotti, Department of Pediatrics, Ribeirão Preto Medical School, University of São Paulo. Gordon Allen Finley, Department of Anesthesiology, Pediatric Pain Research Center, University of Dalhousie, Canada. Correspondence regarding this article should be directed to: Profa Dra Maria Beatriz Martins Linhares, Avenida Tenente Catão Roxo 2650, Prédio da Saúde Mental (salas 52/53), Faculdade de Medicina de Ribeirão Preto, Universidade de São Paulo, Campus Universitário Monte Alegre, Ribeirão Preto, SP, CEP 14048-900, Brasil. Phone: +55-16-36024610. Fax: +55-16-36024504. Email: linhares@fmrp.usp.br that pain is experienced by newborns and children, the translation of scientific knowledge to clinical practice has not occurred to the same degree. Audit studies on the prevalence, assessment, and management of pain in hospitals showed that pain is under-assessed and under-treated in pediatric inpatients (Taylor, Boyer, \& Campbell, 2008; Stevens et al., 2011; Linhares et al., 2012).

Few investments have been made in training professionals and improving clinical practice with regard to pain evaluation and management. Consequently, newborns and children continue to experience unnecessary pain during hospitalization (Karling, Renstrom, \& Ljungman, 2002; Harrison, Loughman, \& Johnston, 2006; Taylor et al., 2008; Stevens et al., 2011).

With regard to health professionals, studies have found problems related to pain evaluation and management. Disagreements exist between the caretakers of patients and children with regard to the evaluation of pain intensity (Elias, Guinsburg, Peres, Balda, \& Santos, 2008; van Dijk, van Wiljck, Kappen, Peelen, Kalkman, \& Schuurmans, 2012). Nurses and physicians report limitations in evaluation tools and methods for pediatric pain management, and pain is 
consequently under-treated during invasive and painful procedures (Chermont, Guinsburg, Balda, \& Kopelman, 2003; Prestes et al., 2005; Kulkamp, Barbosa, \& Bianchini, 2008; Schultz, Loughran-Fowlds, \& Spence, 2010). Professionals also have a lack of knowledge and misconceptions about the prescription of analgesics and opioids (Kulkamp et al., 2008; Daudt, Hadlich, Facin, Aprato, \& Pereira, 1998). Pediatric pain is inadequately managed pharmacologically with regard to scheduling, doses, and intervals (Kulkamp et al., 2008; Daudt et al., 1998). A high incidence of medical mistakes is associated with the prescription of pain medicines (Lerner, Carvalho, Vieira, Lopes, \& Moreira, 2008). Nurses in training and less-experienced physicians (i.e., $<5$ years since undergraduation) have little knowledge about pain (Barros, Pereira, \& Almeida Neto, 2011; Schultz et al., 2010).

Based on the principles of the ChildKind International Initiative (Schechter, Finley, Bright, Laycock, \& Forgeron, 2010), the HC Criança Sem Dor project was initiated at the Hospital das Clínicas at Ribeirão Preto Medical School, University of São Paulo (HCFMRP/USP) involving a collaboration between Brazil and Canada with financial support initially from the Canadian Institutes of Health Research (CIRH). This project was subsequently expanded to the Fernandes Figueira Institute at the Hospital CuidaDOR with support from the Ministry of Health of Brazil.

The first step of the HC Criança Sem Dor project was to conduct a survey to examine the prevalence of pain in 121 hospitalized pediatric patients (i.e., newborns, babies, and children) and the actions taken for pain evaluation and pharmacologic and non-pharmacologic pain management according to the perceptions of patients, families, and health professionals (Linhares et al., 2012). The second step of this project was undertaken in 2011, which was the subject of the present study. We characterized the opinions of health professionals about the evaluation strategies and management of pediatric pain adopted in infirmaries and intensive care units in an educational public hospital. The opinions were related to four axes: (1) pain evaluation, (2) pharmacological management, (3) non-pharmacological management, and (4) continuing education. Each axis included actions, human and material resources, gaps, and needs in different services that cater to hospitalized pediatric patients.

\section{Methods}

\section{Participants}

The sample was composed of 92 professionals who worked in two different units of the HCFMRP/ USP complex (Campus Unit, $n=67$; Emergency Unit, $n=25)$. Health professionals with different pediatric specialties who worked in infirmaries and intensive care units were included, representing the fields of Medicine, Nursing, Psychology, Physiotherapy, and Occupational Therapy. Each professional category had a representative and significant number of participants based on the criterion of directly working with patients or working in coordination to allow decision-making in different services. A professor physician (i.e., service coordinator), two hired physicians, and two residents for each pediatric specialty were included. Head nurses, psychologists, physiotherapists, and occupational therapists who worked in pediatric sectors were also included.

The potential sample for the study included 110 participants, but the sample size decreased by $17 \%$ because 17 physicians and one nurse did not complete the questionnaire. The final sample included 92 health professionals (45 physicians, 18 nurses, 16 psychologists, eight physiotherapists, and five occupational therapists). The professionals' mean time working in their respective field was 10 years $( \pm 9.17$ years). Twenty-seven percent of the sample consisted of service coordinators.

\section{Ethical aspects}

The project was approved by the Research Ethical Committee of Hospital of Clinics, Ribeirão Preto Medical School, University of São Paulo.

\section{Instrument}

For data collection, a questionnaire was developed by two pediatric psychologists and trained researchers in the field of pediatric pain who are members of the Pain in Child Health Program in Canada (i.e., the first and third authors). The final questionnaire was reviewed by a third Canadian researcher with expertise in pediatric pain (sixth author). The questionnaire included 22 open questions that were organized in four axes: pain evaluation, pharmacological management, non-pharmacological management, and continuing education. Each axis included questions related to the following topics: actions, human/material resources, and gaps/needs (see Appendix). The coverpage of the questionnaire presented the goals of the study and instructions for completing the form. The questionnaire was developed based on Strategic Planning principles (Almeida, 2010).

\section{Procedure}

For data collection, the questionnaires were placed in individual envelopes that were personally given to the eligible participants in the study. The questionnaires were self-administered. The researchers gave the respondents 15 days to complete and return the questionnaires.

For data analysis, the participants' responses were categorized based on the quantitative-interpretative method of Biasoli-Alves (1998) for the analysis of thematic content. Two independent researchers applied the categorical system to code the responses. Finally, the data were quantified, organized into database sheets, and analyzed using Statistical Package for Social Sciences software, version 19.0 (Chicago, IL, USA). A descriptive statistical analysis was performed to calculate frequencies and percentages. 


\section{Results}

\section{Sample characteristics}

Table 1 shows that the majority of the professionals belonged to the Campus Unit $(73 \%)$, which has the most pediatric services and beds in HCFMRP/USP. With regard to professional fields, physicians, nurses, and psychologists constituted the majority of the sample $(86 \%)$, which was expected based on the criteria of the composition of the hospital team and consequent study sample.

Table 1. Sample characteristics: hospital units and professional categories

\begin{tabular}{lll}
\hline Sample characteristics & $n$ & \% \\
\hline Hospital Units & & 73 \\
HC - Campus & 67 & 27 \\
HC - Emergency & 25 & \\
& & \\
Profession & & 49 \\
Physician & 45 & 20 \\
Nurse & 18 & 17 \\
Psychologist & 16 & 9 \\
Physiotherapist & 8 & 5 \\
Occupational therapist & 5 & \\
& & \\
\hline
\end{tabular}

Table 2 shows that the professionals worked specifically in different pediatric specialties or pediatrics in general. Sixteen pediatric specialties were included in the study, predominantly neonatology and pediatric intensive care. Table 2 also shows that the majority of the professionals worked in pediatric infirmaries (49\%) or neonatal or pediatric intensive care units $(23 \%)$. The remaining $28 \%$ of the professionals were distributed into different sectors including burn unit $(8 \%)$, child neurology and neurosurgery $(7 \%)$, infectious diseases $(5 \%)$, nursery $(5 \%)$, pediatric surgery $(2 \%)$, and orthopedics $(1 \%)$.

Table 2. Sample characteristics: health professionals in pediatric specialties

\begin{tabular}{lll}
\hline Sample characteristic & $n$ & \% \\
\hline Pediatric Specialty & 17 & 20 \\
General pediatrics & 13 & 15 \\
Neonatology & 12 & 13 \\
Pediatric intensive care & 7 & 8 \\
Burn unit & 6 & 7 \\
Gastroenterology & 5 & 5 \\
Rheumatology & 5 & 5 \\
Neurology & 5 & 5 \\
Infectology & 4 & 4 \\
Endocrinology & 4 & 4 \\
Nephrology & 3 & 3 \\
Oncology & 3 & 3 \\
Pneumology & 2 & 2 \\
Immunology & 2 & 2 \\
Cardiology & 2 & 2 \\
Pediatric surgery & 1 & 1 \\
Orthopedics & 1 & 1 \\
Neurosurgery & &
\end{tabular}

\section{Evaluation of pediatric pain}

Figure 1 illustrates the actions related to pain evaluation. A total of $84 \%$ of the participants reported that they performed pain evaluation in their hospital routine using clinical evaluations (e.g., anamnesis and physical examinations), pain measurement tools or reports, and behavioral observations. Among these methods, the professionals predominantly used clinical evaluations $(32 \%)$. Notably, only $29 \%$ of the professionals reported the use of standard measurement tools (e.g., Faces Scale-Revised, Comfort Scale, Neonatal Facial Coding System Scale, and Neonatal Infant Pain Scale) for pain evaluation, although $43 \%$ of the professionals reported that they had pain measurement tools available in their work sector. Of the respondents, 37\% reported that they did not have materials for pain evaluation or did not know about the existence of these resources.

With regard to gaps in pain evaluation, the professionals highlighted a lack of standardized service protocols and professional training (43\%) in both separate and combined categories, a lack of communication between professionals, and a lack of resources. The lack of human and material resources was reported by $25 \%$ of the professionals. A total of $69 \%$ of the professionals indicated that protocol standardization and training are the main necessities related to the evaluation of pediatric pain.

\section{Pharmacological management}

With regard to pharmacological pain management, Figure 2 shows that the use of medications was mentioned by a majority of the professionals (78\%). Of these, $49 \%$ were aware of the specific type of medication used for pain relief in their work sector.

Complementing these findings, the analysis of only the physicians' responses $(n=45)$ revealed that $47 \%$ reported the use of specific medications for pain in their routine, and $38 \%$ were able to precisely specify the schedule or procedure for prescribing these medications.

With regard to material resources for pharmacological management, the vast majority of the professionals $(91 \%)$ reported that their work sector had pain medications for use with children, and $20 \%$ reported the specific names of the medications that were available by prescription.

Concerning the gaps in the proper management of pediatric pain, the professionals reported a lack of professional training (18\%), difficultly accessing certain medications (14\%), lack of anesthesiologists in clinical pediatric care sectors $(11 \%)$, and lack of standardized service protocols $(8 \%)$. Notably, a high number of responses either ignored these gaps $(13 \%)$ or did not identify them (12\%). This may indicate that some health professionals in the sample did not directly work with medications in their clinical practice (e.g., psychologists, physiotherapists, and occupational therapists).

The professionals' needs were similar to the reported gaps. The professionals highlighted the need for professional training in pain management (26\%), 


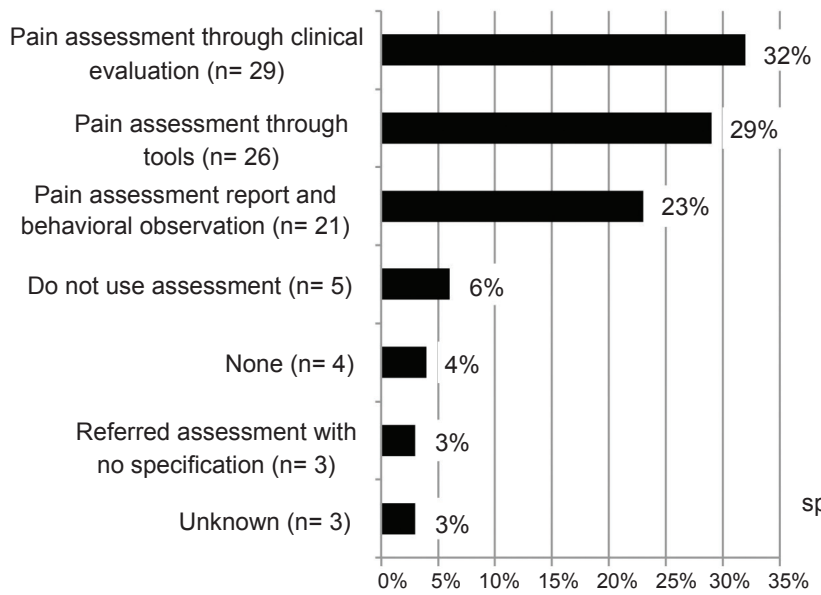

Assessment - Gaps/Barriers

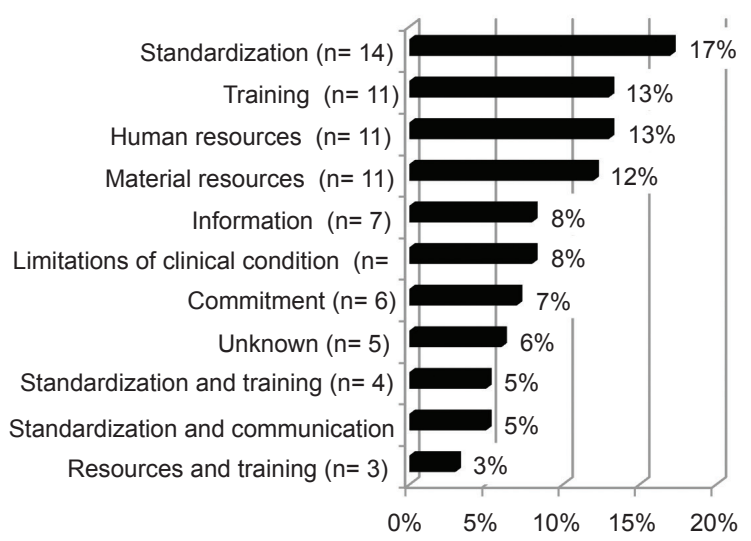

Pain assessment tools $(n=34$

No resources for assesment available $(n=20)$

Monitor or other physiological parameters $(n=8)$

Clinical examination $(n=4)$

Other resources with no specification for assessment $(n=4)$

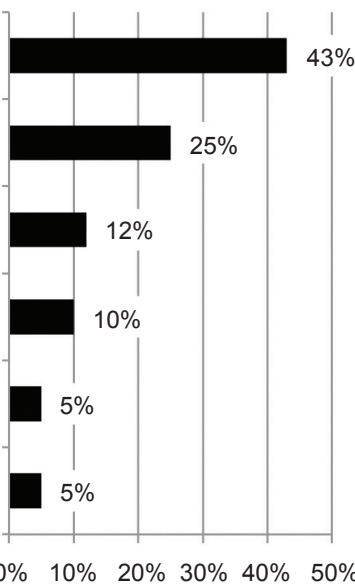

Assessment - Demands

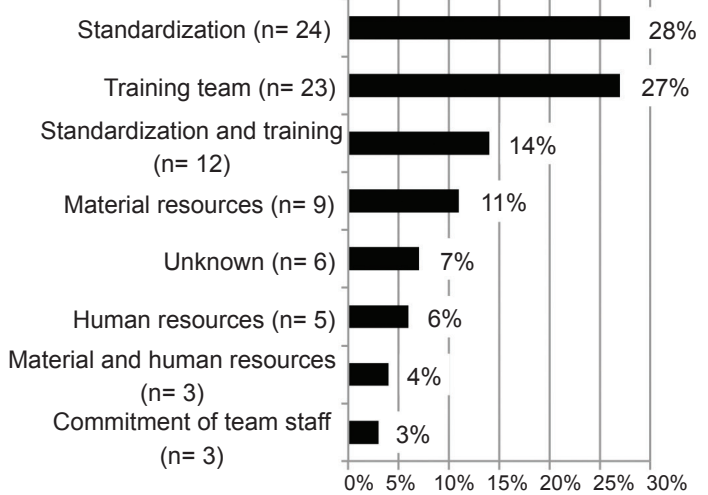

Figure 1. Opinion of health professionals about pain assessment: actions, material resources, gaps/ barriers and demands

standardized pharmacological protocols (22\%), improved accessibility to medications (13\%), and the availability of anesthesiologists in the context of pediatric care and support for invasive and painful procedures $(8 \%)$.

\section{Non-pharmacological management}

With regard to non-pharmacological management actions taken by the professionals, Figure 3 shows that only $25 \%$ of the professionals adopted this type of intervention in the hospital context, and $26 \%$ reported the use of strategies to comfort the patient. Importantly, a high proportion of respondents disregarded or did not use this type of management (18\%). With regard to material resources for non-pharmacological pain management, $65 \%$ of the professionals reported the existence of any resource in their work place. Toys and distraction objects were the most identified resources $(28 \%)$. With regard to gaps, the professionals reiterated a lack of training in the pain field $(25 \%)$, outdated human resources $(22 \%)$, and difficulty communicating and integrating actions between health professional teams $(16 \%)$. The needs reported by the participants followed a similar pattern including human and material resources $(38 \%)$, professional training $(35 \%)$, and the creation of standardized protocols $(10 \%)$.

\section{Discussion}

The goal of the present study was to characterize the opinions of health professionals who work in the hospital context with regard to strategies for the evaluation and management of pediatric pain that are adopted in infirmaries and intensive care units in a public teaching hospital. The main findings showed that few professionals' practices used standardized, validated, and specific tools for pain evaluation. Therefore, the evaluations were predominantly performed using clinical evaluations. Similar findings were reported by Schultz et al. (2010), who analyzed the responses of recently graduated physicians and found that tools for pain evaluation were seldom used in their healthcare routine.

For the evaluation of children, especially newborns who do not verbally communicate, the use of tools that operationalize symptoms of pain is critical. Silva, Balda, \& Guinsburg (2012) analyzed photographs of babies in situations of pain and no pain. They found that medical 


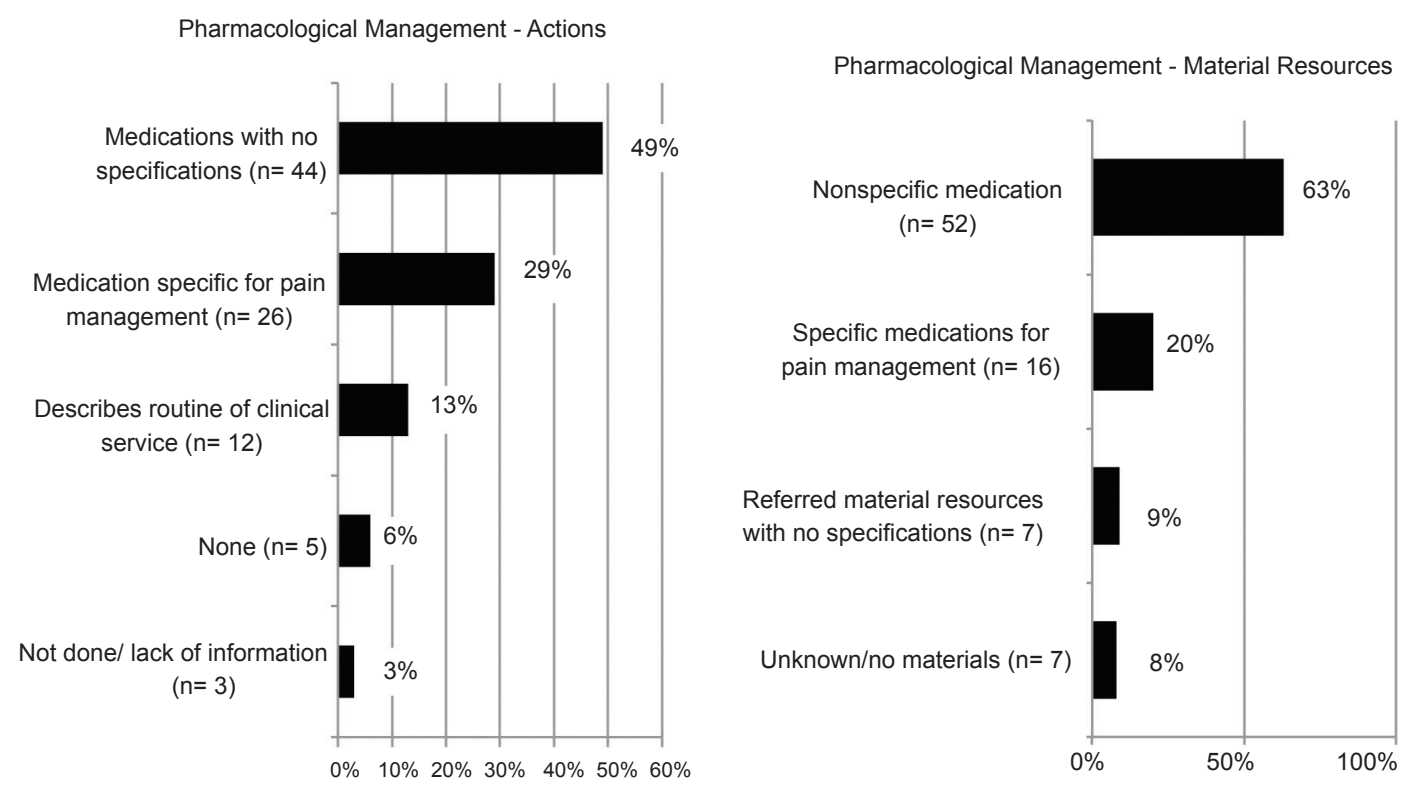

Pharmacological Management - Gaps/Barriers

Pharmacological Management - Demands
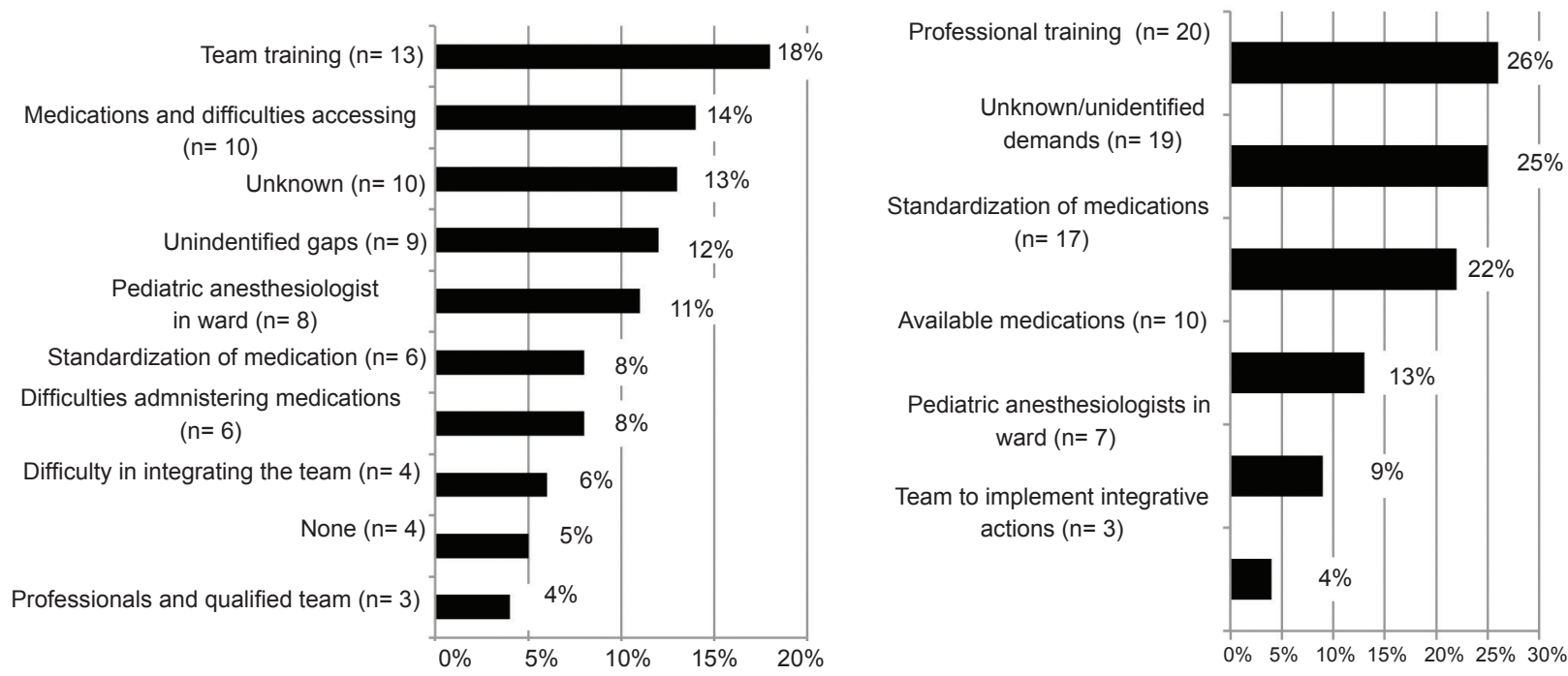

Figure 2. Opinion of health professionals on the pharmacological management of pain: actions, material resources, gaps/ barriers and demands

students and residents in pediatrics and neonatology had difficulty identifying pain in premature infants.

Although clinical examinations help with diagnostic evaluation and the evolution of patients, the use of validated tools specifically for the evaluation of pain has been recommended, such as by the ChildKind Initiative (www.childkindinternational.org; accessed March 2, 2013) that allows the systematization of evaluation protocols. In the area of pain evaluation, published studies have guided the choice of validated tools according to well-established assessments proposed by the PED-IMPACT task force, which performed a critical analysis of evaluation tools in the area of pediatric psychology (Cohen, La Greca, Blount, Kazak, Holmbeck, \& Lemanek, 2008a). The results of this analysis in the clinical context allowed the identification of the use of scales for pain self-reports (Stinson, Kavanagh, Yamada, Gill, \& Stevens, 2006), scales, questionnaires, and daily reports using pain selfmonitoring (Cohen et al., 2008b), and observational measures of pain in children and adolescents (von Baeyer, \& Spagrud, 2007; Cohen et al., 2008b). 


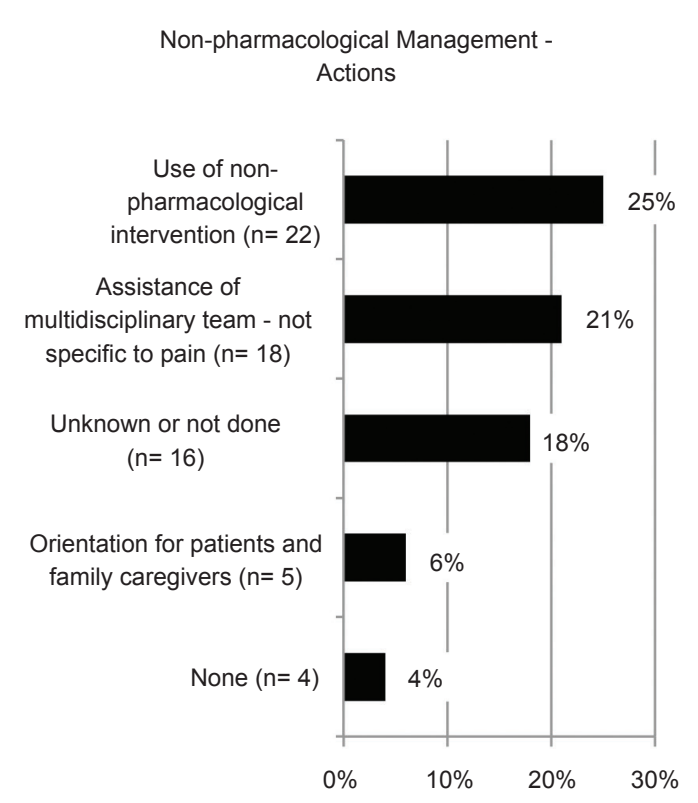

Non-pharmacological Management Gaps/Barriers

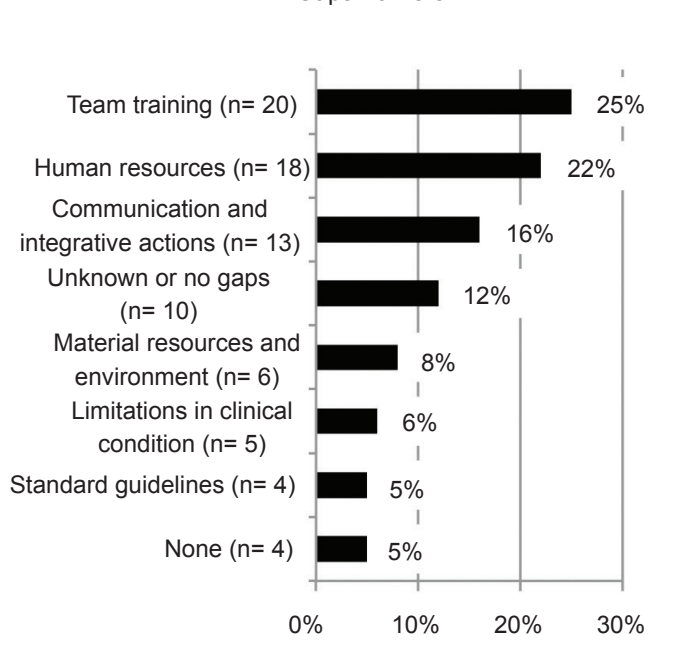

Non-pharmacological Management Material Resources

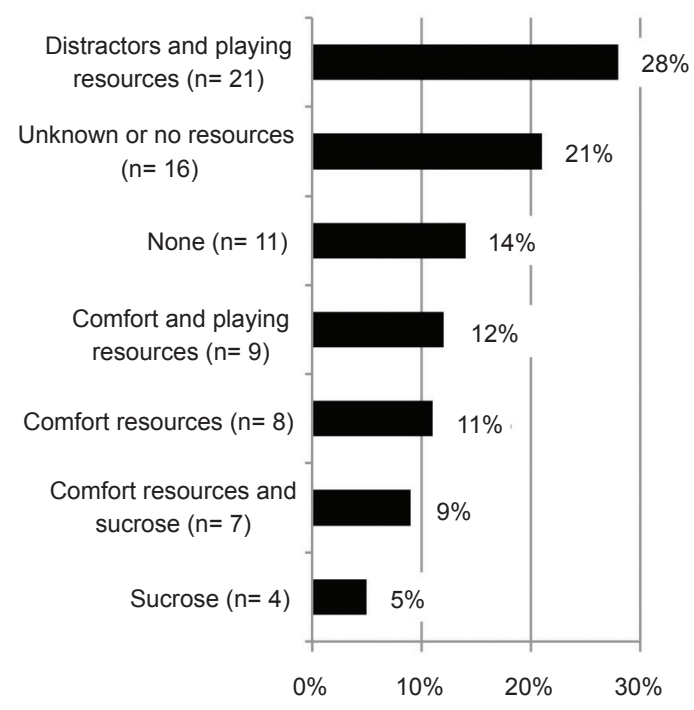

Non-pharmacological Management - Demands

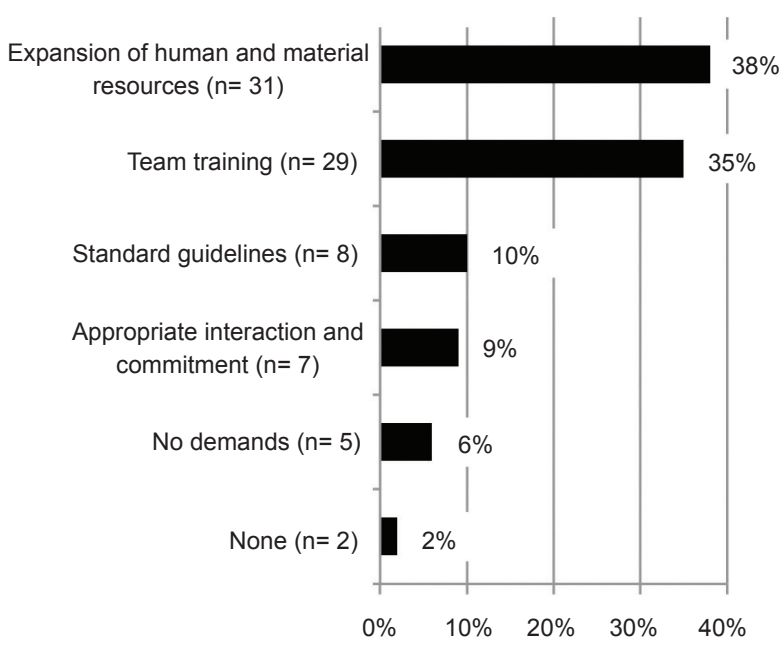

Figure 3. Opinion of health professionals on non-pharmacological management of pain: actions, material resources, gaps/ barriers and demands

A set of well-established instruments is available for pain assessment in infants (e.g., Neonatal Facial Coding System, Premature Infant Pain Profile, and Neonatal Infant Pain Scale) and children (e.g., Faces Pain Scale-Revised, Visual Analog Scale, and the Face, Legs, Activity, Cry, Consolability Scale; Stinson et al., 2006; von Baeyer, \& Spagrud, 2007; Linhares, \& Doca, 2010). However, no "gold standard" for pain evaluation in children and newborns was provided by these studies. They showed that pain needs to be evaluated by tools that combine different behavioral and physiological parameters that complement each other. The combination of report tools with observational measures and evaluations for multiple informants have also been recommended.
The present study found that pain was not systematically evaluated in the evolution of the inpatients. This consequently led to the under-identification and under-treatment of pain. This finding was similar to previous results obtained in both developed countries (Melotti et al., 2005; Taylor et al., 2008; Schultz et al., 2010; Stevens et al., 2011) and developing countries (Chermont et al., 2003; Prestes et al., 2005; Kulkamp et al., 2008; Linhares et al., 2012).

The use of medications for pain relief was reported by $78 \%$ of the health professionals in the present study. However, only $25 \%$ of the health professionals reported that they were aware of non-pharmacological procedures and appropriately specified established and recommended techniques for the purpose of the 
prevention of pain or pain relief in clinical practice (e.g., sucrose, non-nutritive suckling, distraction, and relaxation). Notably, pharmacological interventions were more recognizable by health professionals, and non-pharmacological interventions generated doubts and unfamiliarity about their characteristics, function, and effects in pain management. Even when sucrose was identified as a useful approach for pain relief in newborns, discordance was found among the professionals with regard to its classification as pharmacological or nonpharmacological pain management.

Interventions for pain management must combine both pharmacological and non-pharmacological approaches for pain treatment and prevention, involve training that begins in medical school (Silva, Pinto, Gomes, \& Barbosa, 2011), and involve multidisciplinary teams (Kulkamp et al., 2008). Review studies and guidelines have been published to assist professionals with the choice of pharmacological treatments (Anand et al., 2006) and non-pharmacological management (Anand et al., 2006; Linhares, \& Doca, 2010; Stevens et al., 2011). Management plan recommendations should be based on scientific evidence that can be safely translated to clinical practice.

With regard to the gaps identified by the professionals for the evaluation of pediatric pain in the present study, a lack of specialized professional training and lack of standardized protocols for the evaluation of pain in pediatric services were highlighted. With regard to pharmacological management, a lack of specialized professional training that provides information and aids in the choice of specific medications for pain treatment and lack of standardized protocols for medications in pediatric services were also mentioned. This limited the professionals' knowledge about the pharmacological management of pain, which was also identified by Kulkamp et al. (2008) and Magalhães, Mota, Saleh, Dal Secco, Fusco, \& Gouvêa (2011).

Similarly, a lack of professional training, limited human resources, and little communication and integration between members of different teams who work in this area were also reported for the non-pharmacological management of pain. Silva et al. (2011) studied the perception of pediatric pain from the perspective of nurses and showed that these professionals limited themselves to pharmacological methods, which hindered personal assistance and the quality of care for hospitalized children.

As a result of the reported gaps, most of the professionals in the present study reported the need to implement effective standardized protocols and specialized training for the evaluation and pharmacological management of pain. Moreover, they reported the need for better availability of pediatric anesthesiologists in infirmaries and improved access to medications that are specific for pain relief.

The gaps perceived by the professionals reflected the perception of limitations. This, in turn, reflected the concept of the institutionalization of pain management recommended by the ChildKind Initiative (Schechter et al., 2010). This concept advocates the incorporation of basic principles in the evaluation and treatment of pain in standard daily practice, including systems for documentation and recording, standardized procedures, continuing education for professionals, and quality care programs in institutions that focus on pain management.

With regard to the needs for non-pharmacological pain interventions, the professionals in the present study gave importance to professional training and the implementation of standardized protocols. Moreover, they highlighted the need to expand human and material resources that specifically enable the implementation of these interventions.

In the present study, the health professionals indicated motivation to participate in a program for pain evaluation and pediatric pain management in the hospital. In a study by Magalhães et al. (2011), training was given for the identification, quantification, and treatment of pain in a nursing team. Significantly improved knowledge about the topic was found after training. This is an important initial step, but it does not sufficiently ensure that effective changes in behavior will occur in the clinical practice of pain evaluation and the management of pediatric pain. Continuing education must include in-service procedural training for specialized professionals and the topic "pain" in the academic training of students and residents.

The present study has some limitations. First, it was a descriptive study with the goal of mapping only, without the goal of establishing relationships between variables. Second, the results were based on selfreports and the perceptions of the participants. No direct observations of the professionals' actions were made in the clinical context. Third, the participants' responses were analyzed together, with no professional category discrimination. These points could be addressed in futures studies.

The findings of the present study may establish a baseline for pain evaluation and the management of pain from the perspective of professionals who work in a hospital. The results mapped the prevalence of pediatric pain, adopted actions, and needs reported by the professionals.

\section{Acknowledgements}

The present study was partially supported by the Global Health Research Initiative, Canadian Institutes of Health Research. M.B.M. Linhares participated in conceptualizing, planning, and performing the study, organizing the team for data collection, analyzing and interpreting the results, and preparing the manuscript. N.C.A.C. Oliveira participated in performing the study, collecting the data, analyzing and interpreting the results, and preparing the manuscript. F.N.P. Doca participated in conceptualizing and planning the study and revising the manuscript. F.E. Martinez and A.P. Carlotti participated in collecting the data, analyzing and interpreting the results, and revising the manuscript. G.A. Finley participated in conceptualizing and 
planning the study. All of the authors read and approved the final manuscript. The authors thank the Ministry of Health Hospital Cuidador project for partial financially supporting the study and the CNPq for supporting the research activities of M.B.M. Linhares. The authors also thank the study participants. We also appreciate the support from Pain in Child Health (PICH) in the training of F.N.P. Doca.

\section{References}

Almeida, M. I. R. (2010). Manual de Planejamento estratégico: Desenvolvimento de um plano estratégico com a utilização de planilhas Excel. São Paulo: Atlas.

Anand, K. J. S., Aranda, J. V., Berde, C. B., Buckman, S., Capparelli, E. V., Carlo, W., ... Walco, G. A. (2006). Summary proceedings from the neonatal pain-control group. Pediatrics, 117(Suppl. 1), S9-S22.

Barros, S. R. A. F., Pereira, S. S. L., \& Almeida Neto, A. (2011). A formação de acadêmicos de enfermagem quanto à percepção da dor em duas instituições de ensino superior. Revista Dor, 12, 131-137.

Biasoli-Alves, Z. M. M. (1998). A pesquisa em psicologia: Análise de métodos e estratégias na construção de um conhecimento que se pretende científico. In: G. Romanelli, \& Z. M. M. Biasoli-Alves (Eds.), Diálogos metodológicos sobre prática de pesquisa (pp. 135157). Ribeirão Preto: Legis Summa.

Chermont, A. G., Guinsburg, R., Balda, R. C. X., \& Kopelman, B. I. (2003). O que os pediatras conhecem sobre avaliação e tratamento da dor no recém-nascido? Jornal de Pediatria, 79, 265-272.

Cohen, L. L., La Greca, A. M., Blount, R. L., Kazak, A. E., Holmbeck, G. N., \& Lemanek, K. L. (2008a). Introduction to special issue: Evidence-based assessment in pediatric psychology. Journal of Pediatric Psychology, 33(9), 911-915.

Cohen, L. L., Lemanek, K., Blount, R. L., Dahlquist, L. M., Lim, C. S., Palermo, T. M., ... Weiss, K. E. (2008b). Evidence-based assessment of pediatric pain. Journal of Pediatric Psychology, 33(9), 939-955.

Daudt, A. W., Hadlich, E., Facin, M. A., Aprato, R. M. S., \& Pereira, R. P. (1998). Opióides no manejo da dor: uso correto ou subestimado? Dados de um hospital universitário. Revista da Associação Médica Brasileira, 44, 106-110.

Elias, L. S. D. T., Guinsburg, R., Peres, C. A., Balda, R. C. X., \& Santos, A. M. N. (2008). Discordância entre pais e profissionais de saúde quanto à intensidade da dor no recém-nascido criticamente doente. Jornal de Pediatria, 84, 35-40.

Harrison, D., Loughnan, P., \& Johnston, L. (2006). Pain assessment and procedural pain management practices in neonatal units in Australia. Journal of Paediatrics and Child Health, 42(1-2), 6-9.

International Association for Study of Pain (1997). Curriculum on pain for students in psychology [http://www.iasppain.org/AM/Template. $\mathrm{cfm}$ ? Section=Home $\S$ ion $=$ New_Resources\&template $=/ \mathrm{CM} /$ ContentDisplay.cfm\&ContentFileID $=228$; accessed June 28, 2013].

Karling, M., Renstrom, M., \& Ljungman, G. (2002). Acute and postoperative pain in children: A Swedish nationwide survey. Acta Paediatrica, 91, 660-666.

Kulkamp, I. C., Barbosa, C. G., \& Bianchini, K. C. (2008). Percepção de profissionais da saúde sobre aspectos relacionados à dor e utilização de opióides: Um estudo qualitativo. Ciência e Saúde Coletiva, 13, 721-731.

Lerner, R. B. M. E., Carvalho, M., Vieira, A. A., Lopes, J. M. A., \& Moreira, M. E. L. (2008). Erros medicamentosos em unidade de terapia intensiva neonatal. Jornal de Pediatria, 84, 166-170.

Linhares, M. B. M., \& Doca, F. N. P. (2010). Dor em neonatos e crianças: Avaliação e intervenções não farmacológicas. Temas em Psicologia, 18(2), 307-325.

Linhares, M. B. M., Doca, F. N.P., Martinez, F. E., Carlotti,A.P. P., Cassiano, R. G. M., Pfeifer, ... Finley, G. A. (2012). Pediatric pain: Prevalence, assessment, and management in a teaching hospital. Brazilian Journal of Medical and Biological Research, 45(12), 1287-1294.

Magalhães, P. A. P., Mota, F. A., Saleh, C. M. R., Dal Secco, L. M., Fusco, S. R. G., \& Gouvêa, A. L. (2011). Percepção dos profissionais de enfermagem frente à identificação, quantificação e tratamento da dor em pacientes de uma unidade de terapia intensiva de trauma. Revista Dor, 12, 221-225.

Melotti, R. M., Samolsky-Dekel, B. G., Ricchi, E., Chiari, P., Di Giacinto, I., Carosi, F., \& Di Nino, G. (2005). Pain prevalence and predictors among inpatients in a major Italian teaching hospital: A baseline survey towards a pain free hospital. European Journal of Pain, 9(5), 485-495.

Prestes, A. C., Guinsburg, R., Balda, R. C., Marba, S. T., Rugolo, L. M., Pachi, P. R., \& Bentlin, M. R. (2005). The frequency of pharmacological pain relief in university neonatal intensive care units. Jornal de Pediatria, 81(5), 405-410.

Schechter, N. L., Finley, G. A., Bright, N. S., Laycock, M., \& Forgeron, P. (2010). ChildKind: A global initiative to reduce pain in children. Pediatric Pain Letter, 12(3), 26-30.

Schultz, M., Loughran-Fowlds, A., \& Spence, K. (2010). Neonatal pain: A comparison of the beliefs and practices of junior doctors and current best evidence. Journal of Paediatric and Child Health, 46(1-2), 23-28.

Silva, A. P. M., \& Balda, R. C. X., \& Guinsburg, R. (2012). Reconhecimento da dor no recém-nascido por alunos de medicina, residentes de pediatria e neonatologia. Revista Dor, 13, 35-44.

Silva, M. S., Pinto, M. A., Gomes, L. M. X., \& Barbosa, T. L. A. (2011). Dor na criança internada: A percepção da equipe de enfermagem. Revista Dor, 12, 314-320.

Stevens, B. J., Abbott, L. K., Yamada, J., Harrison, D., Stinson, J., Taddio, A., ... Finley, G. A. (2011). Epidemiology and management of painful procedures in children in Canadian hospitals. Canadian Medical Association Journal, 183(7), E403-E410.

Stinson, J. N., Kavanagh, T., Yamada, J., Gill, N., \& Stevens, B. (2006). Systematic review of the psychometric properties, interpretability and feasibility of self-report pain intensity measures for use in clinical trials in children and adolescents. Pain, 125(1-2), 143-157.

Taylor, E. M., Boyer, K., Campbell, F. A. (2008). Pain in hospitalized children: A prospective cross-sectional survey of pain prevalence, intensity, assessment and management in a Canadian pediatric teaching hospital. Pain Research and Management, 13(1), 25-32.

van Dijk, J. F. M., van Wijck, A. J. M., Kappen, T. H., Peelen, L. M., Kalkman, C. J., \& Schuurmans, M. J. (2012). Postoperative pain assessment based on numeric ratings is not the same for patients and professionals: A cross-sectional study. International Journal of Nursing Studies, 49(1), 65-71.

von Baeyer, C. L., \& Spagrud, L. J. (2007). Systematic review of observational (behavioral) measures of pain for children and adolescents aged 3 to 18 years. Pain, 127(1-2), 140-150. 


\section{Appendix}

\section{Analysis of pediatric care services in the context of hospitalization at HCFMRP/USP}

The goal of this research is to characterize care services at HCFMRP/USP that treat children and adolescents in the context of hospitalization from the perspectives of different health professionals with regard to (1) pain evaluation, (2) pharmacological management, (3) non-pharmacological management, and (4) continuing education. In each axis, we seek to analyze actions, human and material resources, and gaps and needs that exist in different services that cater to hospitalized pediatric patients. We request your special attention and collaboration to complete the attached questionnaire. You do not need to identify yourself by name, but it is important to fill out the characteristics to identify your profession and area of expertise. The data will be analyzed and disclosed to the services so that goals, guidelines, and actions may help reduce the incidence of pain in children. Your experience is crucial for fostering a hospital that takes care of pain in hospitalized children.

Thank you for your collaboration! 


\section{Identification}

Function:

( ) Head of area/clinic ( ) Head of service

Professional area:

( ) Medicine ( ) Nursing ( ) Psychology ( ) Occupational therapy ( ) Physiotherapy Specialty:

Length of time engaged in this function:

Area/Clinic to which you belong:

HC - Campus

$\begin{array}{lll}\text { ( ) Pediatric Infirmary } & \text { ( ) Nursery } & \text { ( ) Pediatric Intensive Care Unit }\end{array}$

( ) Neonatal Intensive Care Unit ( ) Infection Diseases Unit ( ) Oncology
( ) Neurology ( ) Center of Epilepsy ( ) Neurosurgery
( ) Pediatric surgery ( ) Thorax/Cardiovascular ( ) Pediatric Urology
( ) Orthopedics ( ) Other (specify):

HC-Emergency Unit

( ) Infirmary

( ) Burn

( ) Pediatric Intensive Care Unit ( ) Infection Diseases Unit

( ) Other (specify):

Which ACTIONS are functioning in your service, referring to:

Assessment of pediatric pain

What is done?

How is it done?

Pharmacological management of pediatric pain

What is done?

How is it done?

Non-pharmacological management of pediatric pain

What is done?

How is it done?

Continuing professional education on pediatric pain

What is done?

How is it done? (What is the schedule?)

What topics are included?

What is the frequency?

1. What are the HUMAN AND MATERIAL RESOURCES that exist in your service, referring to:

a. Assessment of pediatric pain

b. Pharmacologic management of pediatric pain

c. Non-pharmacologic management of pediatric pain

d. Continuing professional education on pediatric pain

2. What are the GAPS and/or BARRIERS that exist in your service, referring to:

a. Assessment of pediatric pain

b. Pharmacologic management of pediatric pain

c. Non-pharmacologic management of pediatric pain 
d. Continuing professional education on pediatric pain

3. What are the NEEDS that exist in your service, referring to:

a. Assessment of pediatric pain

b. Pharmacologic management of pediatric pain

c. Non-pharmacologic management of pediatric pain

d. Continuing professional education on pediatric pain 